\title{
CORRECTION
}

\section{Correction to: BAL biomarkers' panel for differential diagnosis of interstitial lung diseases}

\author{
Miriana d'Alessandro ${ }^{1}$ (D) Alfonso Carleo ${ }^{2} \cdot$ Paolo Cameli $^{1} \cdot$ Laura Bergantini $^{1} \cdot$ Anna Perrone $^{1} \cdot$ Lucia Vietri $^{1}$. \\ Nicola Lanzarone ${ }^{1}$. Cecilia Vagaggini ${ }^{1}$. Piersante Sestini ${ }^{1}$ - Elena Bargagli ${ }^{1}$
}

Published online: 8 February 2020

(c) Springer Nature Switzerland AG 2020

\section{Correction to: Clinical and Experimental Medicine https://doi.org/10.1007/s10238-020-00608-5}

The original version of this article unfortunately contained a mistake. First and last names of the authors were interchanged. The correct author names are given below.

Miriana d'Alessandro · Alfonso Carleo · Paolo Cameli - Laura Bergantini · Anna Perrone · Lucia Vietri · Nicola Lanzarone · Cecilia Vagaggini · Piersante Sestini · Elena Bargagli

The original article has been corrected.
Publisher's Note Springer Nature remains neutral with regard to jurisdictional claims in published maps and institutional affiliations.

The original article can be found online at https://doi.org/10.1007/ s10238-020-00608-5.

\section{Miriana d'Alessandro}

dalessandro.miriana@gmail.com

1 Respiratory Diseases and Lung Transplantation, Department of Medical and Surgical Sciences \& Neurosciences, Siena University Hospital, University of Siena, Viale Bracci 1, 53100 Siena, Italy

2 Department of Pulmonology, Hannover Medical School, Hannover, Germany 